\title{
Simulation of Surface Instability at the Interface of Two Fluids
}

\author{
Nurdan Yildırım ${ }^{1 *}$, Jean-Marie Buchlin ${ }^{2}$, Carlo Benocci ${ }^{3}$ \\ ${ }^{1}$ Enerji Sist. Müh. Böl, Yaşar Ünv. Bornova-İzmir, +90-232-5708200, +90-232-5707000, \\ nurdan.yildirim@yasar.edu.tr \\ 2 The Von Karman Inst. for Fluid Dynamics, Saint-Genesius-Rode,Belgium, +32-2-3599614, +32-2-3599600, \\ buchlin@vki.ac.be \\ 3 The Von Karman Inst. for Fluid Dynamics, Saint-Genesius-Rode,Belgium, +32-2-3599616, +32-2-3599600, \\ benocci@vki.ac.be \\ * Corresponding author \\ Recieved: $8^{\text {th }}$ February 2017 \\ Accepted: $12^{\text {th }}$ May2017 \\ DOI:10.18466/cbayarfbe.319878
}

\begin{abstract}
Abtract
In this theoretical and numerical study, the physical mechanisms leading to the production of surface waves generated at the interface of two fluids (liquid/gas or liquid/liquid) are investigated. Particular attention is devoted to the Kelvin-Helmholtz $(\mathrm{KH})$ type instability, which appears in the area of high shear located at the fluid-fluid interface. The subsequent disturbances in velocity and pressure associated with the wave motion are assumed to be $2 \mathrm{D}$ and sufficiently small to justify the linearization of the equations of the motion. The Navier-Stokes (NS), Orr-Sommerfeld (OS) and KH equations are the primary ones used to investigate of surface instability. During the study, the main characteristics of a surface instability such as wavelength, wave number, frequency, amplitude, wave speed and growth rate are investigated according to fluid viscosity. The effect of gravity is investigated by 2D simulations without these external forces or with them in one of the following configurations:

- Gravity field from the lighter fluid to heavier fluid

- Gravity field in the co-fluid direction

- Gravity field in the counter co-fluid direction

Fast Fourier Transform (FFT) analysis provides the values of the dominant frequency and wavelength. The wavelength is coupled when the fluids are in the linear instability regime according to the KH-Darcy (KHD) theory. Wave speed of the horizontal flow is determined in the range $0.025-0.04 \mathrm{~m} / \mathrm{s}$ with numerical simulation, and it is determined theoretically to be $0.024 \mathrm{~m} / \mathrm{s}$. This validation shows that the results of the numerical simulation are promising according to the theory.
\end{abstract}

Keywords: - Surface wave, surface instability, viscosity, gravity, two-layer fluid flow.

\section{Introduction}

Liquid film instabilities are encountered in many industrial processes such as strip painting; the production of paper, photographic film and magnetic tape; and packaging. Usually, the liquid film should be thin, continuous, uniform in thickness and smooth. For many years the Von Karman Institute for fluid dynamics (VKI) has been deeply involved in research on liquid film behaviour. One of the previous instability studies of VKI is the Ozgen's Ph.D. thesis from 1999 titled "Two-Layer Flow Instability in Newtonian and non-Newtonian Fluids" 1999 [1]. The other study about instability is Rossi's diploma course (DC) project from 2004. The topic of the project was "Numerical modelling of Gas-Jet Wiping" [2]. In this study, the interaction of the air jet and the liquid film has been investigated numerically using arbitrary fluids. During the study, many difficulties were observed for the numerical modelling of gas-jet wiping. Therefore, this paper is a more fundamental study of the free surface flows where the initial surface-wave generation and 
growth are triggered by the action of the wind. This last mechanism is the most well-known and obvious example of air-sea interaction. The subject has been intensively studied since the pioneering theoretical model proposed by Phillips [3], Benjamin [4], and Miles [5], among others. Nevertheless, neither of the predicted growth rates by these models, in particular those for the short wind waves in the gravity-capillary region, agrees with the experimental measurements. It was not until the analysis of Miles [6], in which the instability of an air shear flow over the water surface was considered, that reasonable growth rates for the gravity-capillary waves were obtained. Miles's analysis did not include the shear flow in the water; it is known that the phase speeds of wind-generated gravity-capillary waves depend on the wind-induced drift in the water. Thus, the analysis was further improved by taking into account the shear flow in the water by Valenzuela [7], Kawai [8] and Wheless and Csanady [9].

Valenzuela [7] was among the first to study the growth of instability waves on the air-sea interface by numerically solving the full formulation of the OS equation and the interfacial conditions for the coupled air-water shear flow [10]. The numerical results of Valenzuela [7] demonstrated that the shear flow in the water can produce a significant increase in the growth of wind-generated gravity-capillary waves. Later, Kawai [8] conducted intensive studies on the initial generation and evolution of the instability waves through both theoretical analyses and laboratory measurements. He found that the frequencies, growth rates and phase velocities of the most unstable waves from the stability analysis are virtually coincident with those of the observed initial wavelets in the laboratory experiments. He therefore concluded that the generation of the initial wavelets at the air-sea interface is caused by selective amplification of the most unstable waves in the coupled air-water shear flow. Wheless and Csanady [9] returned to the full formulation of the instability wave problem, the same as that in the investigations of Valenzuela [7] and Kawai [8], with focuses on studying the internal structure of the instability waves and extending the range of flow parameters explored. They developed a numerical technique to integrate the coupled OS equation based on the compound matrix method, a numerical technique that combines the inviscid and viscid solutions through the use of a Ricatti transformation. They nevertheless claimed that the developed computational method could not cope with the near-discontinuity in the second derivative of the mean air-flow profile characterizing the linear-logarithmic distribution used in most of the previous investigations. For this reason, they constructed a new smooth time-averaged velocity profile with a continuous second derivative. The calculated growth rates that Wheless and Csanady [9] obtained, however, are almost a factor of two larger than those calculated by Kawai [8], who used a linearlogarithmic air velocity profile. They therefore attributed the large discrepancies to the differences in velocity profiles [11]. Cao et al. [12] studied flow instability at the interface of a two-layer, densitymatched, viscosity-stratified Poiseuille flow. They observed that, for the linear case, the interfacial mode is neutrally stable. The growth rate is proportional to the Reynolds number (Re) for small Re and increases with viscosity ratio. Dong and Johnson [13] investigated the instabilities of a channel Coutte flow composed of two layers of immiscible fluids, experimentally and theoretically. Their results show that depending on the depth ratio, the interfacial instability of two-layer fluid flow can be a long wave or a short wave instability, respectively. In the short wave instability regime, the experimental results agree well with the theoretical predictions of the linear instability analysis. They observed that increasing the viscosity of both layers makes the system more stable and that decreasing the density of both layers makes the system more stable. The linear analysis of the KH instability of a cylindrical interface when there is heat and mass transfer across the interface has been conducted by Awasthi et al. [14]. They used an irrotational theory known as viscous correction for the viscous potential flow theory in which the discontinuities in the irrotational tangential velocity and shear stress are eliminated in the global energy balance by taking viscous contributions to the irrotational pressure. It has been shown that the irrotational viscous flow with viscous correction gives rise to exactly the same dispersion relation as obtained by the dissipation method in which the viscous effect is accounted for by evaluating the viscous dissipation using the irrotational flow. Fernandino and Ytrehus [15], Fielding and Wilson [16], Cheung and Zaki [17], and Tzotzi and Andritsos [18] also studied the surface 
instabilities of a two-layer fluid flow.

This study is a fundamental study of the instability that occurs when a gas jet impinges on a moving liquid film (commonly called "jet wiping"). In this study, surface waves, which are generated by a shear flow over a liquid pool, are investigated in 2D. The interaction of two-phase flow (liquid/gas or liquid/liquid) is studied. To remove the wall effects, the middle part of the liquid pool is considered for the study. During the study, the main characteristics of a surface instability such as wavelength, wave number, frequency, amplitude, wave speed and growth rate are investigated according to various flow directions (horizontal, vertical) and fluid viscosities.

\section{Theoretical Background}

The most important equation for this study is the OS Equation. This is the fundamental differential equation governing stability, derived independently by Orr (1907) and Sommerfeld (1908).

Because the problem is linear, it can be investigated by the time evolution of the individual harmonic components:

$$
\psi(x, y, t)=f(y) e^{-i(k x-\omega t)}
$$

where $\mathrm{k}$ is wave number and $\omega$ is the disturbance frequency. The wave speed $\mathrm{c}$ is defined as:

$$
c=\omega / k
$$

For incompressible flow, the OS equation is given in Eq. (2.3).

$$
(U-c)\left(\frac{d^{2} f}{d y^{2}}-k^{2} f\right)-\frac{d^{2} U}{d y^{2}} f=-\frac{i v}{k}\left(\frac{d^{4} f}{d y^{4}}-2 k^{2} \frac{d^{2} f}{d y^{2}}+k^{4} f\right)
$$

Eq. (2.3) can be written in non-dimensional form as Eq. $(2.4)[1,19,20]$ :

$$
(U-c)\left(\frac{d^{2} f}{d y^{2}}-k^{2} f\right)-\frac{d^{2} U}{d y^{2}} f=-\frac{i}{k \operatorname{Re}}\left(\frac{d^{4} f}{d y}{ }^{4}-2 k^{2} \frac{d^{2} f}{d y^{2}}+k^{4} f\right)
$$

For spatial amplification, the wave number, $\mathrm{k}=\mathrm{k}_{\mathrm{r}}+\mathrm{ik}_{\mathrm{i}}$, is complex, and the phase velocity, c, is real in Eq. (2.1). It is convenient to define the frequency of the wave as $\omega=\mathrm{k}_{\mathrm{r}}{ }^{*} \mathrm{c}$. Therefore, Eq. (2.1) can be rewritten as:

$$
\psi(x, y, t)=f(y) e^{\left(-k_{i} x\right)} e^{i\left(k_{r} x-\omega . t\right)}
$$

The spatial growth rate is $-\mathrm{k}_{\mathrm{i}}$, for which $\mathrm{k}_{\mathrm{i}}<0$ yields an unstable configuration, $\mathrm{k}_{\mathrm{i}}=0$ yields a neutrally stable configuration, $\mathrm{k}_{\mathrm{i}}>0$ yields a stable configuration.

The OS equation remains as given in Eq. (2.4), except that $\mathrm{c}$ is replaced by $\omega / \mathrm{k}_{\mathrm{r}}$. The eigenvalue relation is given as $\mathrm{k}=\mathrm{f}(\mathrm{w}, \mathrm{Re})$.

For temporal amplification, the normal modes are still given as in Eq. (2.1) but this time the wave number, $\mathrm{k}$, is real and the wave velocity, $\mathrm{C}=\mathrm{Cr}_{\mathrm{r}}+\mathrm{ic}_{\mathrm{C}}$, is complex. Here, $\mathrm{cr}_{\mathrm{r}}$ is the phase speed and $\mathrm{ci}_{\mathrm{i}}$ is the temporal amplification factor. The frequency, $\omega$, is given as $\omega=k^{*} \mathrm{Cr}$. Thus, normal modes can be rewritten as:

$$
\psi(x, y, t)=f(y) e^{\left(k c_{i} t\right)} e^{i k\left(x-c_{r} t\right)}
$$

Temporal growth rate of the waves for which

$$
\sigma=k c_{i}
$$

$\mathrm{C}_{\mathrm{i}}>0$ yields an unstable configuration,

$C_{i}=0$ yields a neutrally stable configuration,

$\mathrm{c}_{\mathrm{i}}<0$ yields a stable configuration.

The OS equation remains as given in Eq. (2.4). The eigenvalue relation is given as $\mathrm{c}=\mathrm{f}(\mathrm{k}, \mathrm{Re})$. Because the eigenvalue $\mathrm{c}$ appears linearly in the temporal form of the differential equation, most of the work reported in the literature is concentrated on this case. Spatial modes seem to describe the observed instability of parallel flows more faithfully than temporal modes, although their use has not been justified mathematically in an entirely convincing or complete way [1]. The $\mathrm{KH}$ instability can be observed in natural phenomena, for example in clouds In this instability theory, two layers of fluids are considered, one above other, flowing in the same direction but with different velocities $U_{1}$ and $U_{2}$. The fluids can be different densities $\rho_{1}$ and $\rho_{2}$ with the lighter above $\left(\rho_{1}<\rho_{2}\right)$.

Viscosity is not taken into account in $\mathrm{KH}$ instability theory. A second order dispersion relation for the complex celerity of the waves was found as:

$$
c^{2}-2 \frac{\rho_{1} U_{1}+\rho_{2} U_{2}}{\rho_{1}+\rho_{2}} c+\frac{\rho_{1} U_{1}^{2}+\rho_{2} U_{2}^{2}}{\rho_{1}+\rho_{2}}-\frac{\Delta \rho g+\gamma k^{2}}{\left(\rho_{1}+\rho_{2}\right) k}=0
$$

The solutions of Eq. (2.8) are: 


$$
c=c_{r}+i c_{i}
$$

where $\mathrm{Cr}_{\mathrm{r}}$ is the phase velocity of the waves.

$$
c=\frac{\rho_{1} U_{1}+\rho_{2} U_{2}}{\rho_{1}+\rho_{2}} \pm i\left[\frac{\rho_{1} \rho_{2}}{\left(\rho_{1}+\rho_{2}\right)^{2}}\left(U_{1}-U_{2}\right)^{2}-\frac{\Delta \rho g+\gamma k^{2}}{\left(\rho_{1}+\rho_{2}\right) k}\right]^{[1 / 2]}
$$

The wave velocity appears as the mean, weighted by the densities of the two liquid velocities. The condition for the velocity difference $\Delta U=U_{1}-U_{2}$ at which waves grow is

$$
\Delta U^{2} \geq \frac{\rho_{1}+\rho_{2}}{\rho_{1} \rho_{2}}\left(\Delta \rho \frac{g}{k}+\gamma k\right)
$$

The minimum value corresponds to the critical wave number $k_{c}=1 / l_{c}$, where $l_{c}=\sqrt{\frac{\gamma}{\Delta \rho g}}$ is the capillary length. The corresponding minimum velocity threshold is found using Eq. (2.11) at $\mathrm{k}=\mathrm{k}_{\mathrm{c}}$.

Finally, the minimum velocity threshold is

$$
\Delta U_{\min }=\left[\frac{2\left(\rho_{1}+\rho_{2}\right)}{\rho_{1} \rho_{2}}(\Delta \rho g \gamma)^{1 / 2}\right]^{1 / 2}
$$

For $\Delta U \geq \Delta U_{\mathrm{min}}$, the unstable waves are those of numbers in a range containing $\mathrm{k}_{\mathrm{c}}$.

Maximum and minimum wave numbers are calculated as.

$$
k_{\max / \text { min }}=\frac{1}{2}\left\{\frac{\rho_{1} \rho_{2}}{\left(\rho_{1}+\rho_{2}\right)} \frac{(\Delta U)^{2}}{\gamma} \pm\left\{\left(\frac{\rho_{1} \rho_{2}}{\left(\rho_{1}+\rho_{2}\right)} \frac{(\Delta U)^{2}}{\gamma}\right)^{2}-\frac{4 \Delta \rho g}{\gamma}\right\}^{1 / 2}\right]
$$

The growth rate of the instability $\sigma$ can be written in a different form below.

$$
\sigma^{2}=\frac{k_{c}^{3} \gamma}{\left(\rho_{1}+\rho_{2}\right)}\left[-\frac{k}{k_{c}}+\frac{2(\Delta U)^{2}}{\left(\Delta U_{\min }\right)^{2}}\left(\frac{k}{k_{c}}\right)^{2}-\left(\frac{k}{k_{c}}\right)^{3}\right]
$$

\begin{tabular}{|c|c|c|}
\hline & Solver & Segregated \\
\hline Solver & $\begin{array}{l}\text { Unsteady } \\
\text { formulation }\end{array}$ & $\begin{array}{l}1^{\text {st }} \text { order-implicit (only } \\
\text { one allowed with vol- } \\
\text { ume of fluid (VOF)) }\end{array}$ \\
\hline Models & Viscous & Laminar \\
\hline
\end{tabular}

\section{Numerical Approach}

In the study, the commercial software Fluent 6.2 [21] is used. All simulations have been performed with the parameters given in Table 1.

Table 1 General properties of the numerical simulations

\begin{tabular}{lll}
\hline & Multiphase & $\begin{array}{l}\text { VOF } \\
\text { Geo-Reconstruct } \\
\text { Courant number: 0.25 }\end{array}$ \\
\hline & $\begin{array}{l}\text { Pressure-Velocity } \\
\text { Coupling }\end{array}$ & PISO \\
Solution & $\begin{array}{l}\text { Discretization- } \\
\text { Controls }\end{array}$ & $\begin{array}{l}\text { Pressure } \\
\text { Discretization- } \\
\text { Momentum }\end{array}$ \\
\end{tabular}

\subsection{Numerical Domain}

The study focuses on the investigation of surface waves at the interface between two fluids. One test case considered in the study is shown in Figure 1.

For the numerical simulation, only a small part of the pool is considered to remove the wall effects and to allow the use of periodic conditions.

To save CPU time, only a small domain is considered. The dimensions are equal to $10-\mathrm{cm}$ length and $1-\mathrm{cm}$ height for the layer of primary fluid and 1-cm for the layer of secondary fluid. pool is considered to remove the wall effects and to allow the use of periodic conditions.

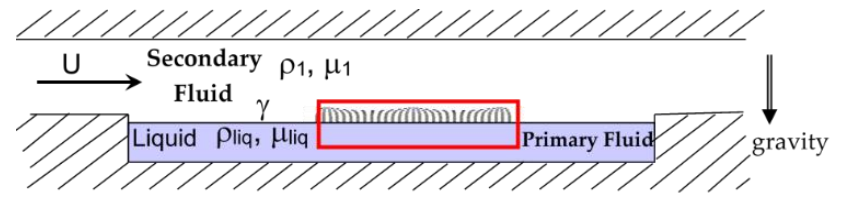

Figure 1 The test case considered in the study.

\subsection{Grids, Time Steps and CPU Time}

Several grids have been considered, including unstructured for the preliminary simulations and structured for the results presented hereafter. Initially, coarse structured meshes were used to check the methodology. It has been found that on the one hand fine, unstructured meshes are costly (on the CPU time view point), and on the other hand, too-coarse meshes do not catch the instabilities. The stretched mesh in the area of the interface is a compromise between low CPU cost and a satisfactory appearance of the instabilities. A close to the interface mesh refinement has been performed, and a fine structure mesh having 8000 cells with $\Delta x=0.5 \mathrm{~mm}$ and $\Delta y \min =0.041 \mathrm{~mm}$ is used for all simulations. The considered grid seems suitable by comparing its $\Delta x$ and $\Delta y$ min distance with the critical wavelength $(16 \mathrm{~mm})$ and capillary length 
(2.56 mm) of water-n-hexane according to the KHD instability theory. The time step of the simulation for water-air is $10^{-6} \mathrm{~s}$ and for water-oil is $10^{-4} \mathrm{~s}$. However, these time steps are the final time steps of the simulations. Because the smaller time steps (i.e., $10^{-8} \mathrm{~s}$ for water-air and $10^{-5} \mathrm{~s}$ for water-oil) are used initially and during the simulations the time steps are increased to prevent explosion of the flow. The CPU time for the simulation is approximately 10 minutes for 100 simulation steps with time step as $10^{-4} \mathrm{~s}$. Some simulations were performed from initial solution to physical time $t=1 \mathrm{~s}$ or even $\mathrm{t}>7 \mathrm{~s}$.

\subsection{Working Fluids}

The density difference between the fluids plays a ma- jor role in the instability of the waves. In Figure 2, instability curves according to KHD theory are shown for different fluid couples. It is clear that the unstable region, which is 'inside' the curve (i.e., to the left of the curve for an observer moving on the curve from the low to high wavelengths), is increasing with decreasing density differences between the fluid couples.

The study investigated water-air and water-oil flows. Initially, water and air were used for the simulations as primary and secondary fluids. The region inside the curve in Figure 2 represents the instability, and the minimum velocity difference to observe some instability is $6.6 \mathrm{~m} / \mathrm{s}$.

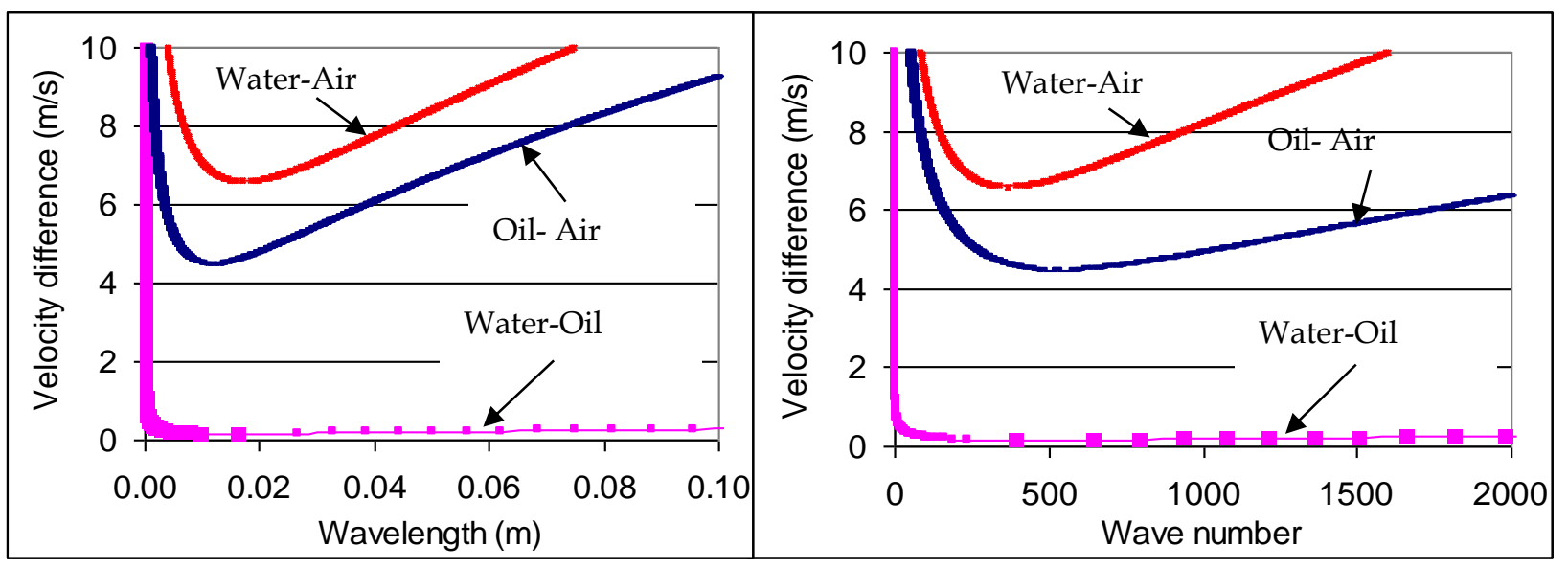

Figure 2 KHD instability curves for different fluid couples.

Therefore, the initial velocities of the air and water are 0 and $7 \mathrm{~m} / \mathrm{s}$, respectively. During the preliminary simulations, it was observed that the heavier fluid has to travel faster than the lighter fluid to keep both converged and physical results consistent.

Water-oil (n-hexane) fluids are used for the simulation as an alternative to water-air because the latter coupled fluid with a high density difference requires a smaller time step to convergence with a standard quality (normalized residuals $=10^{-3}$ for each time step). As seen in Figure 2, the stability curve of water-n-hexane according to KHD presents a minimum velocity difference equal to $0.16 \mathrm{~m} / \mathrm{s}$ to be able to observe instability. This low velocity is associated with a lower difficulty of convergence and with the use of a higher time step for a similar CFL number. On the practical side, it has been observed that the surface instabilities are much faster if water-n-hexane fluids are used for the simulation. Therefore, in this study, mainly watern-hexane flows are used for the simulations with initial velocities of the $n$-hexane and water of 0 and 0.2 $\mathrm{m} / \mathrm{s}$, respectively. The time step is $10^{-4} \mathrm{~s}$. The density of the n-hexane is $660 \mathrm{~kg} / \mathrm{m}^{3}$ and surface tension of the water-oil is taken as $0.0218 \mathrm{~N} / \mathrm{m}$.

\subsection{Boundary Conditions}

Various boundary conditions were applied in the preliminary computations. The use of periodic boundary conditions in the streamwise direction with 0 pressure gradient for a forcing term $(\Delta \mathrm{P}=0)$ is the best option for the study. To avoid wall effects (loose energy through friction), symmetry conditions are used for the upper and lower edges of the numerical domain. 


\section{Post Processing}

For post-processing, mainly FFT, RMS, skewness and flatness analysis have been performed. In addition, growth rates (amplification) of the instability waves have been determined. To be able to make these analyses, a spatial snapshot of the interface y position fluctuation is taken at each $0.01 \mathrm{~s}$. The interface is determined as the line where the volume fraction is equal to 0.5 .

\subsection{RMS, Skewness and Flatness}

The instantaneous root mean square (RMS) of the spatial y position fluctuation indicates the positive deviation of the points according to mean value of the fluctuation distribution. It is calculated as

$$
R M S=\sqrt{\frac{1}{N} \sum_{i=1}^{N}\left(y_{i}-y_{\text {mean }}\right)^{2}}
$$

where $\mathrm{N}$ is the number of points in space.

The variation of this spatial RMS in time will help us to judge the interface from a smooth one to a very wavy one.

The skewness of a distribution indicates the asymmetry of the distribution around its mean, characterizing the shape of the distribution. It is given by:

$$
\text { Skewness }=\frac{\frac{1}{N} \sum_{i=1}^{N}\left(y_{i}-y_{\text {mean }}\right)^{3}}{R M S^{3}}
$$

A positive value of skewness indicates that the distribution is skewed towards values greater than the mean and a negative value indicates that the distribution is skewed towards values smaller than the mean.

$$
\text { * Flatness }=\frac{\frac{1}{N} \sum_{i=1}^{N}\left(y_{i}-y_{\text {mean }}\right)^{4}}{R M S^{4}}
$$

The kurtosis of a distribution indicates the flatness of the distribution with respect to the Gaussian distribution. It is given by

A value of kurtosis higher than 3 indicates that the distribution is flatter than a normal distribution, and a smaller value indicates a higher peak relative to the normal distribution around the mean value [22]. On the top of these indicators, the history of the position maximum of the interface together with minimum and the average value are registered and analysed.

\subsection{Friction Velocity}

The friction velocity of the lighter fluid at the interface is calculated by Eq. (4.4).

$$
{ }^{*} U_{\tau}=\sqrt{\frac{\tau}{\rho_{\text {lighter }}}}=\sqrt{\frac{\left.\mu_{\text {lighter }} \frac{d U}{d y}\right|_{\text {interface }}}{\rho_{\text {lighter }}}}
$$

\subsection{Wave Speed}

A cross correlation method is used to get an idea of wave speed. The replacement for the wave crest is determined by the help of a cross correlation method. According to Alexakis et al. [23] wave speed of the surface gravity waves is determined by

$$
c=\frac{\sqrt{g \lambda A}}{2 \pi}
$$

where

$$
\begin{aligned}
& \mathrm{g} \text { is the gravitational acceleration, } \\
& \lambda \text { is the perturbation mode wavelength } \\
& \mathrm{A} \text { is the Atwood number }
\end{aligned}
$$

The Atwood number for a density of an interface between fluids of density $\rho_{1}$ [upper fluid] and $\rho_{2}$ [lower fluid] with $\rho_{1}<\rho_{2}$ is determined as.

$$
A \equiv \frac{\left(\rho_{2}-\rho_{1}\right)}{\left(\rho_{2}+\rho_{1}\right)}
$$

\section{Results}

To account for the effect of gravity, four simulations have been performed according to the gravity direction. Two additional simulations have been performed to account for the effect of the viscosity. The dimensionless numbers associated to the study are the Weber (We), Reynolds (Re) and Capillary (Ca) numbers. These numbers are determined for the primary and secondary fluids of each simulation by using the critical wavelength, $16 \mathrm{~mm}$, of water-n-hexane according to the KHD instability theory for horizontal flow. Results are given in Table 2.

Table 2 Dimensionless number of the primary and secondary fluid of the simulations.

\begin{tabular}{ccc}
\hline $\begin{array}{c}\text { Dimensionless } \\
\text { Number }\end{array}$ & $\begin{array}{c}\text { Primary } \\
\text { Fluid }\end{array}$ & $\begin{array}{c}\text { Secondary } \\
\text { Fluid }\end{array}$ \\
\hline $\mathrm{We}$ & 29.3 & 0 \\
$\mathrm{Re}$ & 3184.7 & 0 \\
$\mathrm{Ca}$ & 0.009 & 0 \\
\hline
\end{tabular}




\subsection{Effect of the Gravity}

The studied simulations are in the gravity effect section named with the following acronyms:
- $\mathrm{HF}$
- VFAG
- VFSDG
: Horizontal flow
- FWG
: Vertical flow against gravity
: Vertical flow same direction gravity
: Flow without gravity

The common properties of the simulations are given in Table 3.

Table 3 The common properties of the effect of the gravity simulations.

\begin{tabular}{lcc}
\hline \multirow{2}{*}{ Fluids } & $\begin{array}{c}\text { Primary } \\
\text { Fluid }\end{array}$ & $\begin{array}{c}\text { Secondary } \\
\text { Fluid }\end{array}$ \\
\cline { 2 - 3 } & Water & n-hexane \\
\hline Density $\left(\mathrm{kg} / \mathrm{m}^{3}\right)$ & 998.2 & 660 \\
\hline Viscosity $\left(\mathrm{kg} / \mathrm{ms}^{-1}\right)$ & 0.001003 & 0.0003172 \\
\hline Initial Velocity $(\mathrm{m} / \mathrm{s})$ & 0.2 & 0 \\
\hline $\begin{array}{l}\text { Surface Tension } \\
(\mathrm{N} / \mathrm{m})\end{array}$ & \multicolumn{2}{c}{0.021} \\
\hline
\end{tabular}

While the direction of the flow of the fluids is always along $+x$ direction, the direction of gravity will be changed to study the gravitational effect on the formation of wave. The density and viscosity values are taken from the Fluent database. The surface tension value is commonly found for the coupled fluid.

\subsubsection{Horizontal flow (HF)}

In this simulation, the direction of gravity is taken from the lighter fluid to the heavier $(-y)$ and the gravity value is equal to $9.81 \mathrm{~m} / \mathrm{s}^{2}$. The time step is $10^{-4} \mathrm{~s}$. The simulation is performed until the time reached 7.4 s. In other words, the simulation range is $100-74000$ time steps, and the data are collected per 100 time steps.

In Figure 3, the interface contours of the simulation are shown for different times. The interface is created with a volume fraction ratio of water of 0.5 . While initially the interface is smooth, after time $0.1 \mathrm{~s}$ the start of the surface instability can be observed.

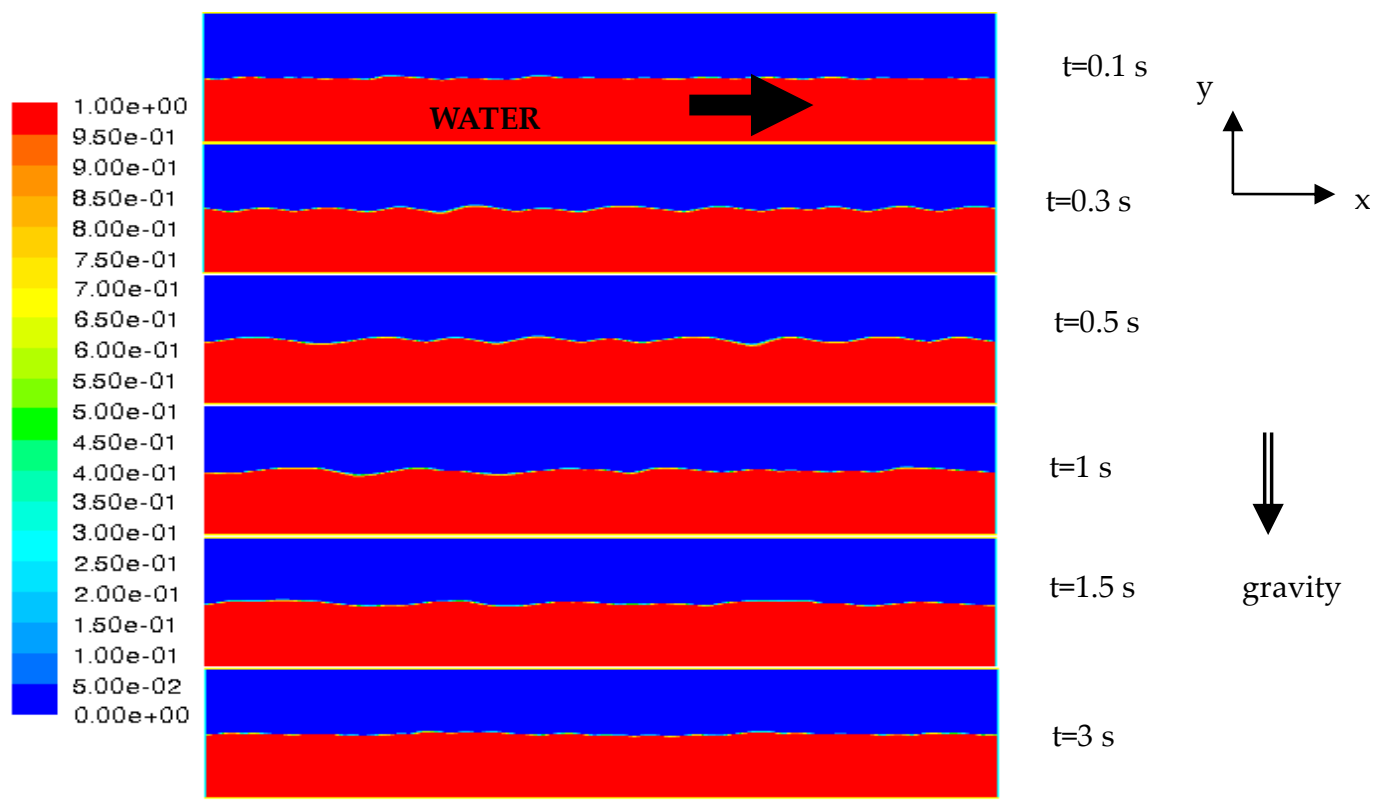

Figure 3 Interface contours of the simulation of horizontal flow.

In Figure 4, the maximum, minimum and mean values of the interface are shown for the time range 0-7.4 s.
According to Techet [24] waves start to be non-linear when the following criterion is met: 


$$
\frac{h}{\lambda}=\frac{2 a}{\lambda}>\frac{1}{7}=0.14286
$$

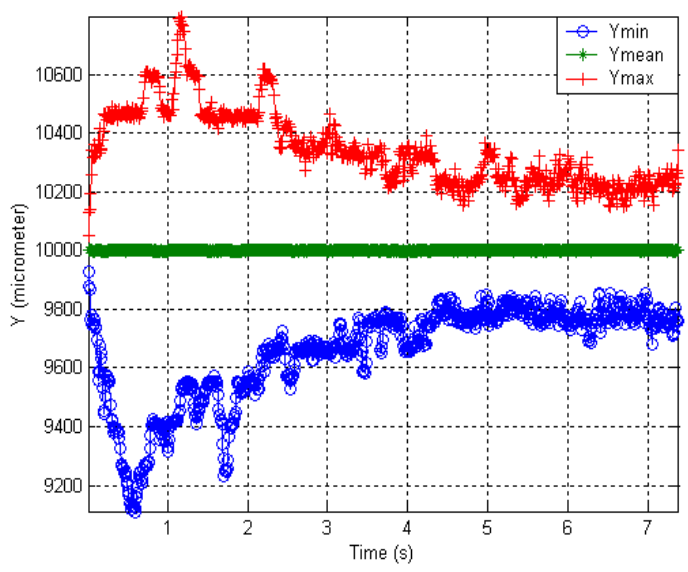

Figure 4 Maximum, minimum and mean values of the interface of horizontal flow.

where $h$ represents twice the wave amplitude (a). $\lambda$ is wavelength. In our case, the maximum amplitude and wavelength are $0.7992 \mathrm{~mm}$ and $11.11 \mathrm{~mm}$, respectively.
As seen in Figure 4, the waves continue to grow until time $1.1688 \mathrm{~s}$. The waves start to be non-linear after time $1.1688 \mathrm{~s}$. At this time, the maximum y value (amplitude) of the interface is reached with a value of $y=10.7992$ $\mathrm{mm}$.

Root mean square (RMS), skewness and flatness analyses have been conducted for the simulation. The results are shown in Figure 5.

Maximum RMS value of interface is $328.1 \mu \mathrm{m}$. According to the KHD instability theory for horizontal flow, the critical wavelength of water-n-hexane is $16 \mathrm{~mm}$. To give an idea of the magnitude of maximum RMS, the value of maximum RMS is normalized by the minimum wavelength of the theory. The normalized maximum RMS is 0.0206 . As seen from Figure 5, after $3 \mathrm{~s}$, skewness and flatness values are approximately 0 and 3 , respectively (i.e the instabilities behave as Gaussian perturbations on the interface).
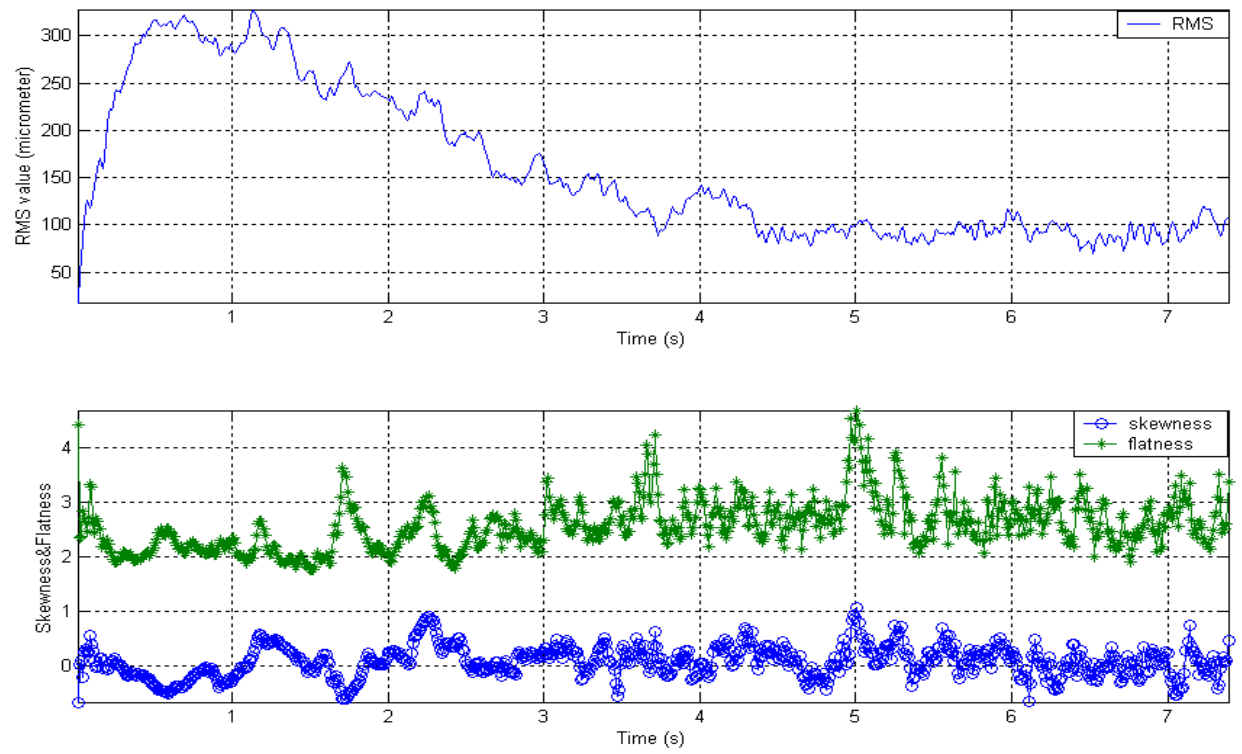

Figure 5 RMS, skewness and flatness analysis of the simulation of horizontal flow.

To get information about frequency and wavelength of the instabilities, FFT analysis has been conducted, and the power spectrum coefficient is calculated through the simulation range (100-74000 time steps) per 100 time steps. The maximum coefficient is $8.8085 \mathrm{e}^{-006}$, and it occurs at time $0.62 \mathrm{~s}$ (6200 time steps). The coefficients are normalized according to the maximum power spectrum coefficient of the simulation and the frequency of the instabilities is considered over a threshold of 0.15

In Figure 6, the Y-level of the interface is shown in the first subplot, the normalized power spectrum coefficient is shown in the second subplot, and the stability 
curves for the velocity differences versus wave number and the wavelength are shown in the third and fourth subplots, respectively, for time $0.62 \mathrm{~s}$.

Figure 6 indicates that the frequencies at time $0.62 \mathrm{~s}$ are 30, 60, 90 and $100 \mathrm{~Hz}$ and they are in the unstable region according to the KHD instability theory. Among the frequencies, $90 \mathrm{~Hz}$ has the maximum coefficient, which means it is the major frequency for time $0.62 \mathrm{~s}$. The wavelength is $11.11 \mathrm{~mm}$.
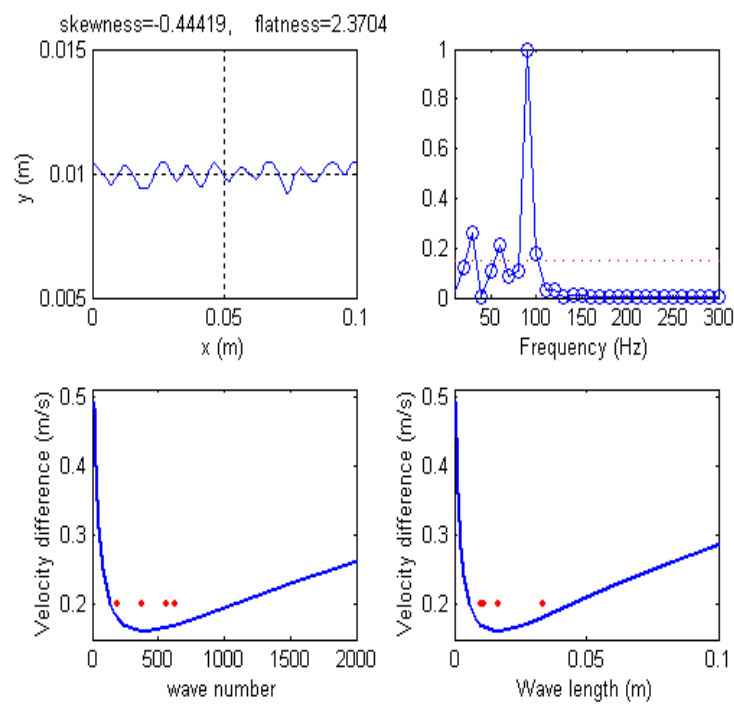

Figure 6 FFT analysis of the simulation of the horizontal flow at time $0.62 \mathrm{~s}$.

During the simulation, there is an exchange of momentum from the moving phase to the steady one with the appearance in time of a boundary layer on each side of the initial interface. In this horizontal simulation, no major numerical artefacts are noticed due to the periodic conditions, which are explained in the section 3.5.

This will not be the case for vertical simulations. In Figure 7 the velocity profiles of $n$-hexane and water at $\mathrm{x}=0.05 \mathrm{~m}$ are shown.

Mean velocity profiles of the fluids are shown in Figure 8. As seen in Figure 8, while the water velocity decreases, the $n$-hexane velocity increases depending on exchange momentum and mass conservation. Through the simulation, the time averages of mean velocity of $n$-hexane and water are 0.063 and 0.157 $\mathrm{m} / \mathrm{s}$, respectively.
The friction velocity of the lighter fluid at the interface is calculated by Eq. 30 to be $6.5 \mathrm{~mm} / \mathrm{s}$ at time

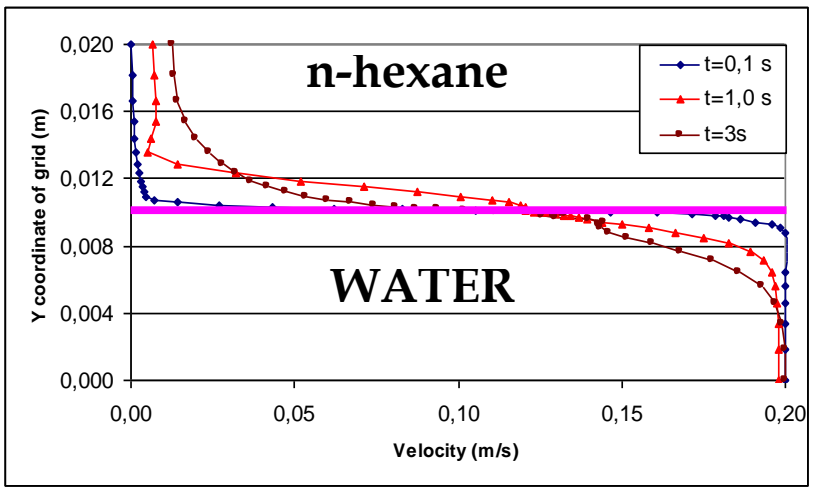

Figure 7 Velocity profiles of n-hexane and water of the horizontal flow simulation for different times.

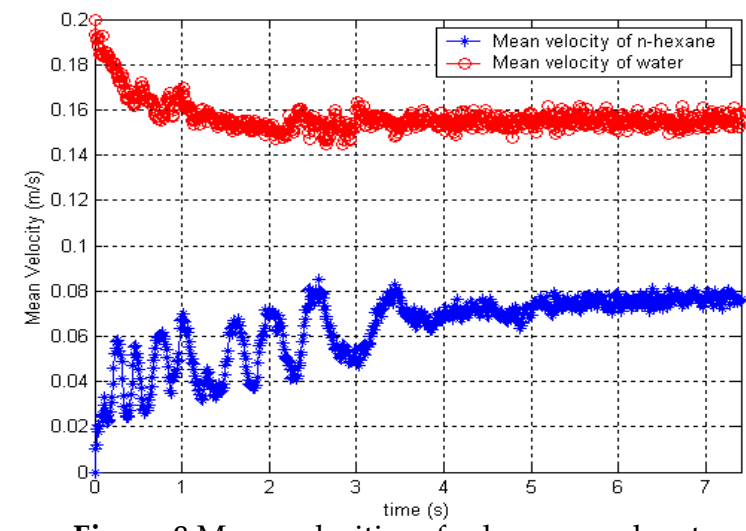

Figure 8 Mean velocities of $n$-hexane and water of the horizontal flow.

$\mathrm{t}=0.62 \mathrm{~s}$. Wave speed of the horizontal flow is determined in the range $0.025-0.04 \mathrm{~m} / \mathrm{s}$, depending on $\Delta x$ of the interface and the time differences among the data in the simulation. In our case, for horizontal flow the Atwood number is determined as 0.204. Wave speed is approximately $0.024 \mathrm{~m} / \mathrm{s}$ with a wavelength of $11.11 \mathrm{~mm}$. This result shows that the results of numerical simulation are compatible with theory according to wave speed.

\subsubsection{Vertical Flow Against to the Gravity (VFAG)}

The study of vertical flows is interesting because of the link with jet wiping investigation. In this simulation, water flows against gravity with $0.2 \mathrm{~m} / \mathrm{s}$ of initial velocity, while n-hexane is initially stationary. The remaining properties are same with HF. In Figure 9(a) the vorticity and interface contours of the simulation 
are shown at time $0.171 \mathrm{~s}$. The figure clearly indicates that the vorticity occurs surrounding the interface. On the other hand, positive and negative pressures can be observed through the interface (Figure 9(b)).

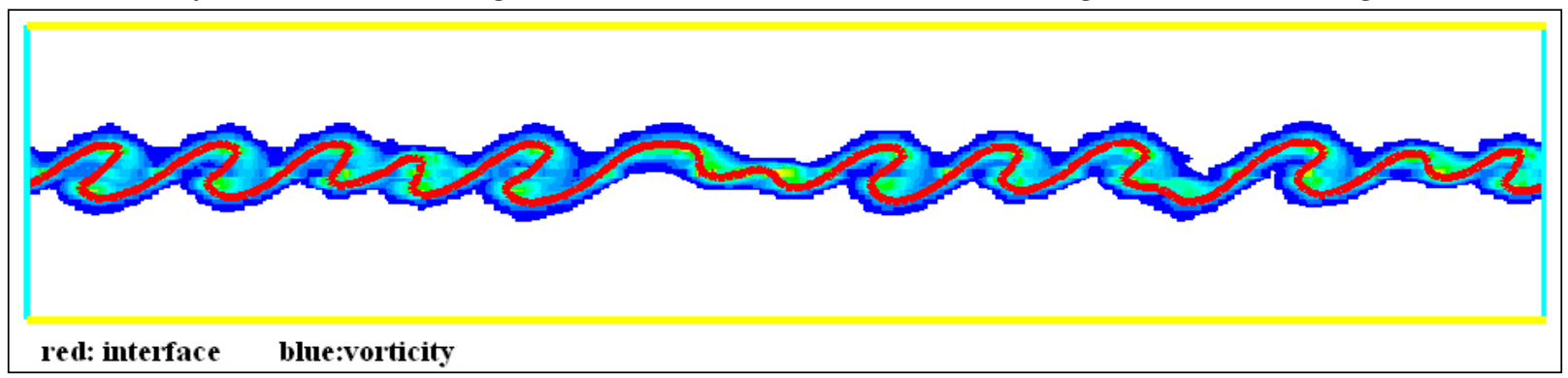

(a)

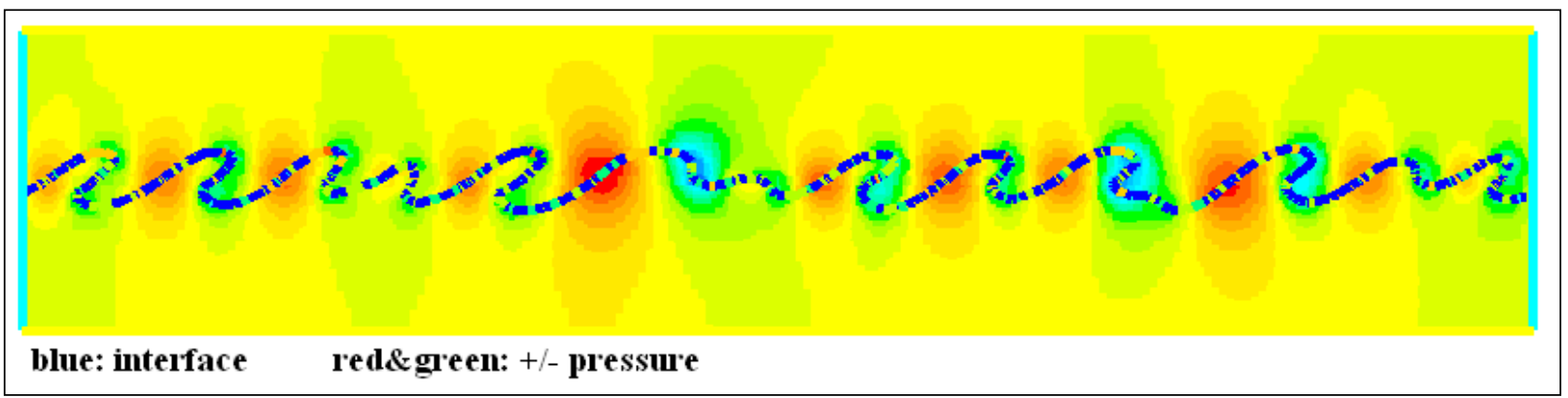

(b)

Figure 9 At time $0.171 \mathrm{~s}$, an illustration of the interface with a) iso-contours of major vorticity and b) iso-contours of the periodic pressure.

\subsection{Effect of Viscosity}

To be able to see effect of the viscosity on the free surface flow, two additional simulations have been performed using an arbitrary fluid with different viscosity. The simulations are:

- HF_HVSF : Horizontal Flow high viscosity secon dary fluid.

- VFSDG_SVSF: Vertical flow same direction gravity small viscosity secondary fluid

In the horizontal flow simulation (HF_HVSF), an arbitrary fluid is used for the secondary fluid with three times higher viscosity than water, $0.003009 \mathrm{~kg} / \mathrm{ms}^{-1}$. The remaining properties are same with HF.

In the vertical flow simulation (VFSDG_SVSF), an arbitrary fluid is used for secondary fluid with 10 times lower viscosity than water, $0.0001003 \mathrm{~kg} / \mathrm{ms}^{-1}$. The other simulation parameters are repeated with the VFSDG simulation.

\subsection{Comparison of the Results}

To give an idea about magnitude, the value of RMS and maximum amplitude of each simulation are nor- malized by the critical wavelength of the theory. According to the KHD instability theory for horizontal flow, the critical wavelength of water-n-hexane is 16 $\mathrm{mm}$.

The key results, such as the main frequency, wavelength, maximum RMS, maximum $Y$ value of interface, mean velocities of the fluids and friction velocity of the gravity and viscosity effect simulations, are given in Table 4.

In Figure. 10, the interface contours of the gravity and viscosity effect simulations are shown. In the simulations, after some time, finger-shaped waves are observed, and post-processing is performed until the finger shapes occur. In vertical flows, the finger-shaped waves are observed very quickly. Therefore, their post-processing time is very short compared to horizontal flow and the flow without gravity. As can be seen from the figure for vertical flows, the direction of the finger shapes of the heavier fluid is always opposite to the gravity direction, whatever the flow direction. 
Table4 Main results of the simulations

\begin{tabular}{llllllll}
\hline Simulations & Unit & HF & VFAG & VFSDG & FWG & HF_HVSF & VFSDG_SVSF \\
\hline Maximum normalized RMS & & 0.0206 & 0.0348 & 0.0313 & 0.1836 & 0.0092 & 0.0297 \\
\hline $\begin{array}{l}\text { Maximum Y value of inter- } \\
\text { face }\end{array}$ & $(\mathrm{mm})$ & 10.799 & 11 & 11.1 & 17.04 & 10.355 & 11.3 \\
\hline Frequency & $(\mathrm{Hz})$ & 90 & 140 & 150 & 30 & 70 & 160 \\
\hline Time for max. frequency & $(\mathrm{s})$ & 0.62 & 0.151 & 0.059 & 0.761 & 0.347 & 0.059 \\
\hline $\begin{array}{l}\text { Time average of mean ve- } \\
\text { locities of n-hexane }\end{array}$ & $(\mathrm{m} / \mathrm{s})$ & 0.063 & 0.335 & 0.172 & 0.056 & 0.039 & 0.142 \\
\hline $\begin{array}{l}\text { Time average of mean ve- } \\
\text { locities of water }\end{array}$ & $(\mathrm{m} / \mathrm{s})$ & 0.157 & 0.185 & 0.273 & 0.170 & 0.162 & 0.278 \\
\hline Wavelength & $(\mathrm{mm})$ & 11.11 & 7.1 & 6.67 & 33.33 & 14.285 & 6.25 \\
\hline Friction velocity & $(\mathrm{mm} / \mathrm{s})$ & 6.5 & 14.7 & 15 & 3.7 & 16.6 & 8.6 \\
\hline
\end{tabular}

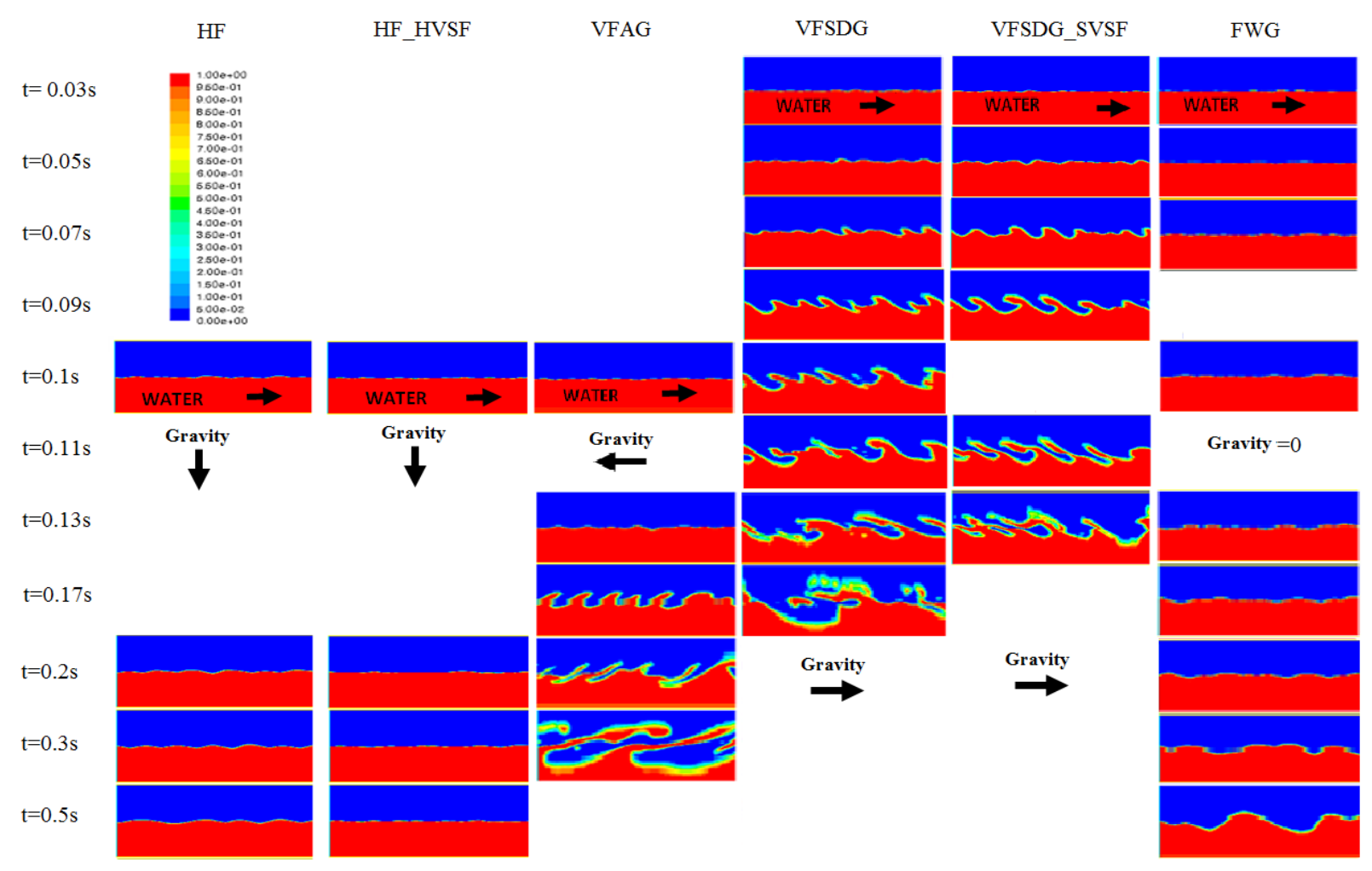

Figure 10 Interface contours of the gravity effect simulations.

Density and fluid velocities play a part in the direction of finger shapes. While the flow direction is against gravity, a lighter fluid has higher velocity; if the flow direction is the same as the gravity direction, the heavier fluid has higher velocity. Therefore, to analyse the direction of the finger shapes, the determination of the phase with the higher velocity is crucial. It is clearly seen that from Figure 10, which compares the results of the HF and HF_HVSF simulations, if the 
secondary fluid has a high viscosity, the amplitude of the instability waves is reduced. As seen in the interface contours (Figure 10), at the initial time, VFSDG has high amplification, but through all simulations, VFSDG_SVSF has the highest values.

\section{Discussions and Conclusions}

In this study, the surface waves generated at the interface of two fluids are investigated in 2D. The main characteristics of the waves such as the wavelength, the wave number, the frequency, the amplitude, the wave speed and the amplification are presented.

The study contains theoretical and numerical approaches. Numerical simulations have been performed by using a VOF multiphase model of the commercial software Fluent 6.2. NS, OS and KH equations are mainly considered to theoretically investigate surface instabilities.

In the paper, the effect of dynamic viscosity has been studied. Additionally, the gravitational effect on the fluids is investigated. While direction of the flow of the fluids is always along $+x$ direction, the gravity direction is changed to study the gravitational effect on the formation of a wave. In the vertical cases, the oscillations are quickly transformed in finger shapes that are sheared before being broken in droplets. Postprocessing is stopped when the finger shapes occur because in this case, for a given position in $\mathrm{x}$, several positions in $y$ of the interface may occur. The results of the simulations show that, for vertical flows, the direction of the finger shapes (in the heavier phase) is always opposite to the gravity direction, whatever the flow direction. For the horizontal flow, the waves are followed from the linear regime to the completely non-linear one. According to the literature, a wave enters the non-linear regime when the ratio between the amplitude (peak to peak) and the wavelength is over $1 / 7$. In our simulation and according to the different indicators (RMS, FFT), the waves for horizontal flow enter in the non-linear regime after a time equal to $1.1688 \mathrm{~s}$. This observation from the numerical simulations is compatible with prediction given by the literature. While the waves continue to grow until time $1.1688 \mathrm{~s}$, the predominant frequency of the simulation is observed at time $0.62 \mathrm{~s}$ according to FFT analysis. After this time, the energy contained in this dominant frequency is distributed to the other wavelengths and the non-linear regime is quickly reached. Wave speed of the horizontal flow is determined in the range $0.025-0.04 \mathrm{~m} / \mathrm{s}$ by using a cross correlation method for the results of the numerical simulation, whereas it is theoretically determined to be $0.024 \mathrm{~m} / \mathrm{s}$. This validation shows that the results of numerical simulations are promising according to the theory for wave speed.

The wavelengths provided by FFT analysis are compared with the KHD instability theory according to an initial velocity difference of $0.2 \mathrm{~m} / \mathrm{s}$. Despite difficulties in extracting the FFT content, the results compare well with the theory. Even if the theory is established only for horizontal flow, it has been used for vertical flows and the numerical instability region always fits with theory. This methodology seems to be adequate to predict the unstable region for the case where the theory is not yet available.

Finally, as a recommendation for further work, it has been observed that the use of periodic boundary conditions in the streamwise direction with zero pressure gradient $(\Delta \mathrm{P}=0)$ and the gravity field in the same direction produced a numerical artefact leading to 'inversion' of velocity (the phase with the lowest velocity may become the phase with the higher velocity depending of its density and the direction of the flow compare to the gravity). To avoid this problem, an individual periodic boundary condition by phase should be implemented. This individual periodic boundary condition would consider the primary and secondary fluid separately while keeping a constant mass flow rate of each fluid during the simulation.

As a suggestion for an easier study of the surface instabilities, the choice of fluid couples with small density difference is advised. Unfortunately, the targeted application (jet wiping of air on liquid zinc) has a density difference of $6540 \mathrm{~kg} / \mathrm{m}^{3}$ [2] and is mainly concerned with vertical up flow, so a proper control of the different phases is necessary.

\section{Acknowledgments}

The author would like to thank the Von Karman Institute for Fluid Dynamics (VKI); this study is a part of the project carried out in VKI titled "Simulation of Surface Instability of a Liquid Pool Subjected to a 
Shear Flow" [25].

\section{REFERENCES}

[1]. Ozgen, S. Two-Layer Flow Instability in Newtonian and non-Newtonian Fluids, Universite Libre de Bruxelles and Von Karman Institute, Ph.D. Thesis, 1999.

[2]. Rossi, V. Numerical Modelling of Gas-Jet Wiping, Von Karman Institute, Project Report 17, 2004.

[3]. Philips, O.M. On the generation of waves by turbulent wind. Journal of Fluid Mechanics. 1957; 2, 417.

[4]. Benjamin, T.B. Shearing Flow Over a Wavy Boundary. Journal of Fluid Mechanics. 1959; 6, 161.

[5]. Miles, J.W. On the generation of surface waves by shear flows. Journal of Fluid Mechanics. 1957; 3, 185.

[6]. Miles, J.W. On the generation of surface waves by shear flows. Part 4. Journal of Fluid Mechanics. 1962; 13, 433.

[7]. Valenzuela, G.R. The Growth of Gravity-Capillary Waves in a Coupled Shear Flow. Journal of Fluid Mechanics. 1976; 76, 229-250.

[8]. Kawai, S. Generation of Initial Wavelets by Instability of a Coupled Shear Flow and Their Evolution to Wind Waves. Journal of Fluid Mechanics. 1979; 93, 661-703.

[9]. Wheless, G.H.; Csanady, G.T. Instability Waves on the Air-Sea Interface. Journal of Fluid Mechanics. 1993; 248, 363-381.

[10]. Lock, R. C. Hydrodynamic stability of the flow in the laminar boundary layer between parallel streams. Proccedings of Cambridge Philosophical Society. 1954; 50, 105-124.

[11]. Tsai, W.T.; Lin, M.Y. Stability Analysis on the Initial Surface-Wave Generation Within an Air-Sea Coupled Shear Flow. Journal of Marine Science and Technology. 2004; 123, 200-208.

[12]. Cao, Q.; Sarkar, K.; Prasad, A.K. Direct numerical simulations of two-layer viscosity-stratified flow. International Journal of Multiphase Flow. 2004; 30, 1485-1508.

[13]. Dong L.; Johnson, D. Experimental and theoretical study of the interfacial instability between two shear fluids in a channel Couette flow. International Journal of Heat and Fluid Flow. 2005; 26, 133-140.
[14]. Awasthi, M.K.; Asthana, R.; Agrawal, G.S. Viscous correction for the viscous potential flow analysis of KelvinHelmholtz instability of cylindrical flow with heat and mass transfer. International Journal of Heat and Mass Transfer. 2014; 78, 251-259.

[15]. Fernandino, M.; Ytrehus, T. Determination of flow subregimes in stratified air-water channel flow using LDV spectra. International Journal of Multiphase Flow. 2006; 32, 436-446.

[16]. Fielding, S.M.; Wilson, H.J. Shear banding and interfacial instability in planar Poiseuille flow. Journal of NonNewtonian Fluid Mech. 2010; 165, 196-202.

[17]. Cheung L.C.; Zaki, T.A. A nonlinear PSE method for two-fluid shear flows with complex interfacial topology. Journal of Computational Physics. 2011; 230, 6756-6777.

[18]. Tzotzi C.; Andritsos N. Interfacial shear stress in wavy stratified gas-liquid flow in horizontal pipes. International Journal of Multiphase Flow. 2013; 54, 43-54.

[19]. Apsley, D. D. Instability and Transition, University of Manchester, School of Mechanical, Aerospace and Civil Engineering http://personalpages.manchester.ac.uk/staff/david.d.apsley/lectures/turbbl/stability.pdf (access in August, 2014)

[20]. Van den Borre, G. Wind Induced Instabilities on a Thin Layer of Aaircraft de or Anti-Icing Fluid. Von Karman Institute, Project Report 32, 1999.

[21]. Fluent 6.1 Documentation, http://jullio.pe.kr/fluent6.1/help/ (access in August, 2014)

[22]. Anthoine, J. Advanced Data Processing. Von Karman Institute. Course notes 2005.

[23]. Alexakis, A.; Young, Y.; Rosner, R. Shear Instability of Fluid Interfaces: Stability Analysis, Physical Review E. 2002; $65,026313$.

[24]. Techet, A. Free-Surface Waves, Massachusetts Institute of Technology, Ocean Engineering, Department of Mechanical

http://web.mit.edu/13.012/www/handouts/Free-Sur-

face\%20Waves_note.pdf (access in August, 2014)

[25]. Yildirim, N. Simulation of Surface Instability of a Liquid Pool Subjected to a Shear Flow. Von Karman Institute, Project Report, 2005 